\title{
Körperliche (In)Aktivität in Kindheit und Jugend
}

\section{U. Walter ${ }^{1}$, S. Kramer ${ }^{1}$, M. Röbl ${ }^{2}$}

${ }^{1}$ Medizinische Hochschule Hannover, Prävention und Rehabilitation in der System- und Versorgungsforschung an der Abt. Epidemiologie, Sozialmedizin und Gesundheitssystemforschung 2Universitätskinderklinik Göttingen, Pädiatrie II

\section{Physical (in)activity in childhood and adolescence}

In the last two decades in Germany and other countries physical activity of children and adolescents declined while sedentary lifestyle increased. Physical inactivity in childhood and youth is related with coronary heart disease risk factors and higher prevalence of obesity. International recommendations suggest daily 60 minutes or more moderate to vigorous physical activity and less than 2 hours sedentary activity in leisure.

eingereicht: 28.9.2005 akzeptiert: 9.11.2005

\section{Aktueller Trend}

Internationale und nationale Studien zeigen eine Abnahme der alltäglichen Bewegung und der körperlichen Leistungsfähigkeit von Kindern und Jugendlichen. Ein Review von Bös weist eine Verminderung der motorischen Leistungsfähigkeit in den vergangenen 25 Jahren um mehr als 10\% aus (Motorische Leistungsfähigkeit von Kindern und Jugendlichen. Erster Deutscher Kinder- und Ju2003). Eine britische Studie zeigt einen Rückgang (1985-1992) der per Fuß oder Fahrrad zurückgelegten Wege bei 0-14-Jähgendsportbericht. Universität Karlsruhe

rigen um 20\% bzw. 26\%(BMJ 1997; 314: 710). Nach einer schottischen Studie sind 3-5-Jährige lediglich 20-25 Min./Tag mäßig bis intensiv körperlich aktiv. 76\% der Zeit verbringen sie mit einer sitzenden Tätigkeit (Lancet 2004; 363: 211-212). Die Verringerung der alltäglichen Bewegung trägt zu einer Reduktion der gesamten körperlichen Aktivität und zu Übergewicht bei Kindern bei. Gortsmaker et al. und ähnlich Crespo et al. beobachteten eine Dosis-Wirkungs-Beziehung zwischen Übergewicht und Fernsehkonsum (Arch Pediatr Adolesc Med 1996; 150: 356-362; Arch Pediatr Adolesc Med 2001; 155(3): 360-365). Hernandez et al. er-

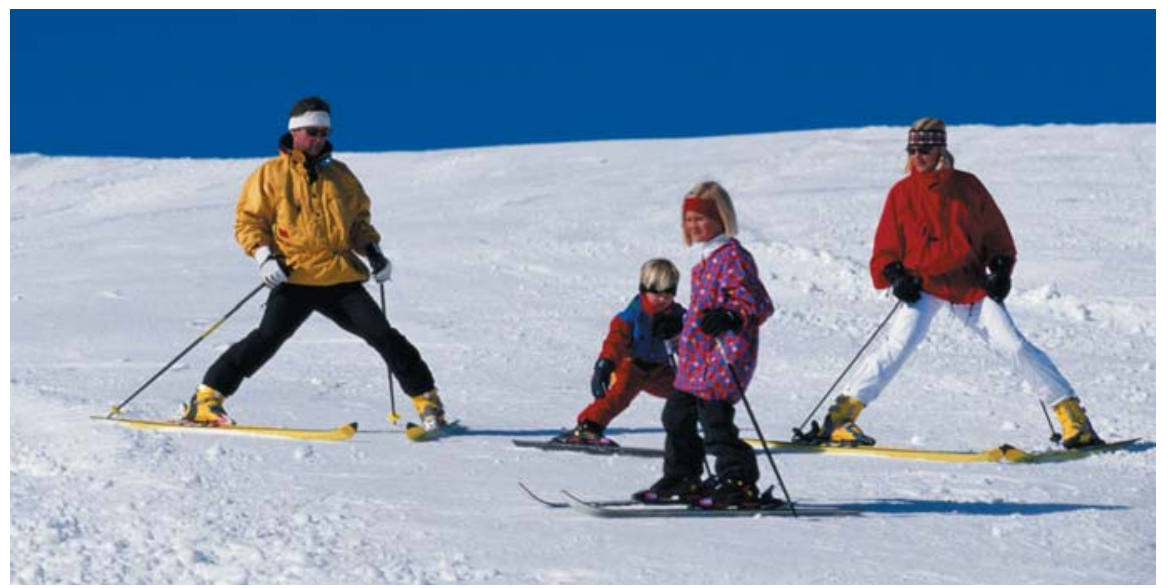

Früh übt sich: Wer Bewegung und Sport schon in der Kindheit lernt, ist auch als Erwachsener eher sportlich aktiv (Bild: Photodisc). mittelten bei 9-16-Jährigen eine Assoziation zwischen Übergewicht, körperlicher Aktivität und Fernsehen (Int J Obes Relat Metab Disord 1999; 23(8): 845-854).

Übergewichtige Kinder sind deutlich weniger körperlich aktiv als Normalgewichtige (Int J Obes Relat Metab Disord 2001; 25(6): 822-829). Sowohl quer- als auch längsschnittliche Beobachtungsstudien zeigen eine geringere Prävalenz von Adipositas bei körperlich sehr aktiven Jugendlichen als bei weniger Aktiven (Pedia 2005; 146(6): 732737).

\section{Situation in Deutschland}

Nach der Studie „Health Behavior in School-aged Children" (HBSC) (22 EU-Länder und USA) befinden sich 11-, 13- und 15-Jährige in Deutschland hinsichtlich der regelmäßigen körperlichen Aktivität im unteren Drittel, nur 18\% der Mädchen und 29\% der Jungen sind an mindestens 5 Tagen der Woche körperlich aktiv (Gesundh-Wes 2005; 67(6): 422431).

Dem Projekt „Fit sein macht Schule" liegt der in mehreren Bundesländern eingeführte „Bewegungs-Check up" zugrunde. Die Auswertungen der Jahre 2001-2003 zeigen einen deutlichen Rückgang der körperlichen Fitness bei 9-15-Jährigen hinsichtlich anaerober Ausdauer, Koordination und Kraft. Von den 6-10-Jährigen ist ein Viertel max. einmal wöchentlich sportlich aktiv, ein Drittel absolviert ein (fast) tägliches Sportpensum. Insgesamt sind Jungen körperlich aktiver als Mädchen. Mit zunehmendem Körpergewicht nimmt bei beiden Geschlechtern die körperliche Aktivität wie auch die Mitgliedschaft in Sportvereinen ab. Von vermehrtem Schulsport (mind. 3 Std./Woche) profitieren bezüglich einer Verbesserung der motorischen Leistungsfähigkeit, wie Auswertungen bei 11-15-Jährigen zeigen, insbesondere Mädchen („Fit sein macht Schule" 2004. Bonn, AOK Bundesverband, Deutscher Sportbund (DSB), Wissenschaftliches Institut der Ärzte Deutschlands (WIAD)).

Folgen körperlicher (In)Aktivität

Die nationale Langzeitstudie „Growing Up Today" zeigt von 1997-1998 bei 11900 1015-jährigen Jungen und Mädchen, dass eine 
Zunahme körperlicher Aktivität zu einem geringeren BMI und vermindertem Übergewicht führt. Jede Zunahme körperlicher Aktivität um eine Stunde/Tag ist z. B. bei 914-jährigen Mädchen mit einer BMI-Reduktion von $0,03 \mathrm{~kg} / \mathrm{m}^{2}$ assoziiert (Pediatrics 2003; 111(4): 836-843). Ähnlich zeigt ein 10-Jahres-Follow-up bei 18-30-Jährigen eine Abnahme des Körpergewichts bei ansteigender körperlicher Aktivität (Int J Obes 2000; 24: 1475-1487).

Das bevölkerungsbezogene attributable Risiko körperlicher Inaktivität für die Mortalität von koronaren Herzkrankheiten und für Diabetes mellitus beträgt jeweils 35\% (Med Sci Sports Exerc 1994; 26: 851-856). Zur physiologischen Wirksamkeit von körperlicher Aktivität und Fitness sowie zu präventiven Wirkungen auf Mortalität und (Gesamt-)Morbidität liegen zahlreiche Studien vor, die u. a. Einfluss auf koronare Herzkrankheit, Hypertonie, Diabetes mellitus Typ 2, Brust- und Darmkrebs bis hin zu Depression/Angst zeigen (Samitz G, Mensink G, eds. Körperliche Aktivität in Prävention und Therapie. München: Hans Marseille, 2002: 11-31; ACSM - American College of Sports Medicine. ACSM's guidelines for exercise testing and prescription. Baltimore: Lippincott Williams \& Wilkins, 2000; KKH, MHH. Weißbuch Prävention! HERZgesund? Hannover, 2004). Entsprechend den positiven Effekten auf das kardio-pulmonale metabolische System fördert aerobes Ausdauer- sowie Koordinationstraining die kognitive Funktionsfähigkeit, erhöht die Gedächtnisleistung im mittleren Lebensalter (Soc Sci Med 2003; 56: 785-792). Körperliche Aktivität führt neben einer Erhöhung des BNDF („brain derived neurotrophic factor")-Spiegels und anderer Wachstumsfaktoren zu einer Anregung der Genexpression, die Prozesse der Plastizität begünstigt (Trends Neurosci 2002; 25: 295-301).

\section{Nutzen körperlicher Aktivität}

Erste Ergebnisse des deutschen CHILT-Projektes („Children's Interventional Trial") zeigen auch bei Kindern einen positiven $\mathrm{Zu}$ sammenhang zwischen motorischen und kognitiven Leistungen (Dtsch Z Sportmed 2003; 54(9): 242-246). Ein aktiver Lebensstil ist positiv mit motorischer Entwicklung korreliert, Kinder mit Adipositas und Überge- wicht haben eine schlechtere motorische Entwicklung (Int J Obesity 2004; 28(1): 2226). Bereits bei 4-7-Jährigen geht eine höhere physische Aktivität mit niedrigeren kardiovaskulären Risikofaktoren wie BMI, Gesamtcholesterin, HDL/ Gesamtcholesterin-Ratio, Triglyzeride und systolischer Blutdruck einher (Scand J Med Sci Sports 2003; 14: 143-149).

Eine Studie an kanadischen Schülern in der Pubertät zeigte, dass ein 3-mal pro Woche über 2 Jahre durchgeführtes 10-minütiges Zirkeltraining bei Mädchen und Jungen zu einem signifikant höheren Anstieg der Knochendichte im Oberschenkelhals und bei Mädchen zusätzlich in den Lendenwirbelkörpern führte (Pediatrics 2003; 112(1): e447-452; Bone 2004; 34(4): 755-764).

Neben positiven Effekten auf die körperliche Funktionsfähigkeit und physiologische Parameter wirkt sich sportliche Aktivität auch auf die psychische Gesundheit aus. So zeigt eine Untersuchung deutscher 1418-Jähriger deutliche Assoziationen zwischen Ausdauersport und Selbstbild, seelischem Wohlbefinden und geringem Alkohol- und Tabakkonsum (Soc Psychiatry Psychiatr Epidemiol 2002; 37(11): 544-550).

Nach einem systematischen Review ist körperliche Aktivität in allen eingeschlossenen Studien höher bei Männern als bei Frauen und invers assoziiert mit dem Alter (Med Sci Sports Exerc 2002; 34(12): 1996-2001). Konsistente Determinanten sind sozioökonomischer Status, Berufstätigkeit („occupational status"), Bildung (,educational attainment"). Selbstwirksamkeit zur Bewegungsaktivität sowie soziale Unterstützung sind konsistente Korrelate körperlicher Aktivität, ebenso konsistent zeigt sich die nicht vorhandene Assoziation zum Wissen um Gesundheit und körperliche Aktivität. Studien ab Ende der 1990er Jahre zeigen den Einfluss umfeldbedingter Faktoren wie Zugang, gemeindebezogene Einflüsse, häufige Beobachtung körperlicher Aktivität, Ausrüstung sowie anregende Umgebung.

\section{Einfluss bis ins Erwachsenenalter}

Dem Kindesalter und der (Vor-)Pubertät kommt insbesondere bei der Entwicklung von Verhaltensmustern über die Lebenszeit eine entscheidende Bedeutung zu. So beträgt nach dem Alter von 6 Jahren die Wahrscheinlichkeit für Adipositas im Erwachsenenalter $50 \%$ für bereits adipöse und $10 \%$ für nicht-adipöse Kinder. Adipositas im Kindesalter ist damit ein bedeutender Prädiktor für adulte Adipositas, wobei mit zunehmendem Alter der Kinder der Einfluss bestehender Adipositas der Eltern abnimmt ( $N$ Engl J Med 1997; 337(13): 869-873). Ebenso wie Adipositas hat auch in jungen Jahren etablierte körperliche (In)Aktivität eine hohe Wahrscheinlichkeit von Persistenz im Lebensverlauf (Am J Public Health 1994; 84(7): 1121-1126).

Prinzipiell sind drei Beziehungen zwischen körperlicher Aktivität in Kindheit und Jugend und Gesundheit im Erwachsenenalter denkbar (in: Gisolfi C, Lamb D, eds. Perspectives in exercise science and sports medicine. New York: McGraw-Hill, 1989: 605-613; Sports Med 2001; 31(8): 617-627): 1.) Körperliche Aktivität von Kindern und Jugendlichen beeinflusst ihre Gesundheit, die ein wesentlicher Prädiktor für die Gesundheit im Erwachsenenalter ist. 2.) Körperliche Aktivität in Kindheit und Jugend beeinflusst die körperliche Aktivität im Erwachsenenalter, die sich wiederum mit hoher Evidenz positiv auf die Gesundheit auswirkt. 3.) Körperliche Aktivität von Kindern und Jugendlichen wirkt sich direkt auf die adulte Gesundheit aus.

Ein Review von Twisk zeigt insgesamt nur geringe Evidenz für die Auswirkungen körperlicher Aktivität in Kindheit und Jugend auf die Gesundheit im Erwachsenenalter (Sports Med 2001; 31(8): 617-627). Sallis et al. verweisen in ihrem Review auf widersprüchliche Ergebnisse (Med Sci Sports Exerc 2000; 32(5): 963-975). Nach einer über 21 Jahre verfolgten Kohortenstudie, beginnend im Alter von 3 Jahren, ist ein hoher und insbesondere kontinuierlicher Level an körper- 
Zielbereich „Gesund aufwachsen: Ernährung, Bewegung, Stressbewältigung" definiert (www. gesundheitsziele.de - Forum zur Entwicklung und Umsetzung von Gesundheitszielen in Deutschland. Bericht. 2003. Berlin). Bildung und Gesundheit sind eng miteinander verbunden und wechselseitig aufeinander bezogen.

licher Aktivität zwischen 9 und 18 Jahren ein Prädiktor für hohe körperliche Aktivität im zweiten bzw. dritten Lebensjahrzehnt ( $A m \mathrm{~J}$ Prev Med 2005; 28(3): 267-273).

Internationale Empfehlungen

Auf Basis vorliegender Evidenz und unter Berücksichtigung der Praktikabilität von Handlungsorientierungen liegen inzwischen auch einige Empfehlungen für Kinder vor (s. Infokasten 1). Ein systematischer Review über 850 Artikel zu dem evidenzbasierten Einfluss körperlicher Aktivität auf Verhalten und Gesundheit bei 6-18-Jährigen führt ebenfalls zu der Empfehlung täglicher mindestens 60-minütiger moderater bis intensiver körperlicher Aktivität und unter 2 Stunden/Tag sitzende Tätigkeit in der Freizeit. Die körperliche Aktivität sollte die Freude an der Bewegung fördern, an den Entwicklungsstand angepasst sein und helfen, grundlegende sowie spezialisierte motorische Fähigkeiten zu erwerben (Pediatr 2005; 146(6): 732-737).

Umfangreichen Schulprogrammen wird das Potenzial zugeschrieben, den altersbezogenen Rückgang der körperlichen Aktivität zu verlangsamen und Schülern zu helfen, lebenslange gesundheitsfördernde Bewegungsmuster zu etablieren. Zur Ausschöpfung der Ressourcen wird eine Koordination schulischer und kommunaler Maßnahmen empfohlen (MMWR 1997; 46(RR6); Prev Med 1993; 22: 544-560).

\section{Förderung körperlicher Aktivität}

Bewegung bildet ein zentrales Handlungsfeld in der Förderung der Gesundheit der Kinder in Deutschland. Im Rahmen von „gesundheitsziele.de“ wurde die Stärkung der motorischen Fähigkeiten und die Reduktion von Bewegungsmangel als ein Oberziel im

Empfehlungen der „American Heart As-
sociation“ zur Primärprävention arterio-
sklerotischer kardiovaskulärer Erkran-
kungen im Kindesalter:
-mind. 1 Std./Tag moderate bis intensive
körperliche Aktivität
-eine Begrenzung der sitzenden Tätigkei-
ten, insbesondere des Fernsehkonsums
auf max. 2 Std./Tag
(Circulation 2003; 107(11): 1562-1566)

\section{Kindertagesstätte und Schule}

Bewegungsfördernde Maßnahmen sollten im Rahmen des Besuchs von Institutionen wie Kindertagesstätte und Schule durchgeführt werden. Bereits die Bewältigung des Hin- und Rückweges zur Einrichtung zu Fuß oder per Fahrrad ermöglicht zusätzliche Bewegung (s. Infokasten 2). Viele bereits begonnene Initiativen und Programme sind übergreifend. Als curricularer Bestandteil, außerunterrichtliches und organisatorisches Element wird Bewegung u. a. im Konzept der bewegungsfreudigen Schule aufgegriffen (Bewegungsfreudige Schule, Verlag Bertelsmann Stiftung, Gütersloh 2005). Eine Befreiung vom Schulsport sollte nur in indizierten medizinischen Ausnahmefällen erfolgen.

\section{Freizeit}

Weiterhin tragen Angebote der Kommunen (adäquate Spielplätze und Freizeiteinrichtungen, gerade in sozial benachteiligten Stadtteilen) und der Sportvereine zur Steigerung der körperlichen Aktivität in der Freizeit bei. Wichtig sind auch spezielle Sportangebote für übergewichtige Kinder und Jugendliche, um gerade für diesen Personenkreis eine Reduktion der Hemmschwelle zu bewirken. Die Auswahl gemein-

samer familiärer Aktivitäten in der Freizeit sollte ebenfalls der Förderung körperlicher Aktivität Rechnung tragen. Das Ausmaß der körperlichen Aktivität von Kindern und Jugendlichen in ihrer Freizeit wird entscheidend von dem elterlichen Vorbild mitbestimmt, Kinder von körperlich aktiveren Eltern betreiben deutlich mehr Sport (Dtsch Arztebl2003; 100(47):3110-3114). Ohne ein Umdenken der Erwachsenen ist eine Förderung der körperlichen Aktivität von Kindern und Jugendlichen nicht zu erreichen.

\section{Infokasten 2}

Die Förderung der körperlichen Aktivität
von Kindern bietet prinzipiell folgende
Ansatzpunkte:
-gemeinsame Aktivitäten mit den Eltern
-Sportangebote von Kommunen und
Sportvereinen
-als curricularer Bestandteil des
Unterrichts,
-in der Förderung der Schule als
Bewegungsraum
-die Öffnung der Schule für sport-
bezogene Angebote in der Freizeit.

Korrespondenz Prof. Dr. Ulla Walter

Prävention und Rehabilitation in der System- und Versorgungsforschung Stiftungslehrstuhl an der Abt. Epidemiologie, Sozialmedizin und Gesundheitssystemforschung

Medizinische Hochschule Hannover 30623 Hannover

Tel.: 0511/532 4455

Fax: 0511/532 5347

E-Mail:walter.ulla@mh-hannover.de DOI: 10.1055/s-2005-923319

\section{Fachliche Betreuung der „Serie Prävention“:}

Prof. Dr. Dr. h. c. Peter C. Scriba Medizinische Klinik / Klinikum Innenstadt der Universität München

Ziemssenstr. 1

80336 München

Prof. Dr. Friedrich W. Schwartz Medizinische Hochschule Hannover Abteilung für Epidemiologie, Sozialmedizin und Gesundheitsforschung

Carl-Neuberg-Str. 1

30623 Hannover 